Ebisu Ebisu

Études japonaises Études japonaises

47 | printemps-été 2012

Catastrophes du 11 mars 2011, désastre de

Fukushima : fractures et émergences

\title{
La promotion du nucléaire civil dans la presse quotidienne japonaise
}

The Promotion of Nuclear Energy in the Japanese Press

日本の新聞における原子力の宣伝

\section{Tino Bruno}

\section{OpenEdition \\ Journals}

Édition électronique

URL : http://journals.openedition.org/ebisu/320

DOI : 10.4000/ebisu.320

ISSN : 2189-1893

Éditeur :

Institut français de recherche sur le Japon (UMIFRE 19 MAEE-CNRS), Maison franco-japonaise

Édition imprimée

Date de publication : 1 juin 2012

Pagination : 99-106

ISSN : 1340-3656

Référence électronique

Tino Bruno, «La promotion du nucléaire civil dans la presse quotidienne japonaise », Ebisu [En ligne], 47 | printemps-été 2012, mis en ligne le 03 avril 2014, consulté le 01 mai 2019. URL : http:// journals.openedition.org/ebisu/320 ; DOI : 10.4000/ebisu.320 


\title{
La promotion du nucléaire civil dans la presse quotidienne japonaise
}

\author{
Tino BRUNO
}

Alors que l'accident de la centrale nucléaire Fukushima Daiichi commence à redessiner les contours d'une société japonaise de nouveau traumatisée par l'atome, et à l'heure où le Japon traverse sa " plus grave crise depuis la Seconde Guerre mondiale" ", tous les regards sont portés sur sa politique énergétique.

Grand projet politique d'un Japon des années 1950 voulant s'autonomiser d'un point de vue énergétique alors que sa croissance économique était fulgurante, le nucléaire fut promu avec faste alors que l'occupation américaine touchait à sa fin et que le président américain de l'époque, Dwight D. Eisenhower, eut prononcé son fameux discours «Atoms for Peace ${ }^{2}$ ». Émissions radiophoniques puis télévisuelles, articles dans la presse, livres, films, produits de consommation courante, le nucléaire s'invitait même dans l'événementiel et les parcs de loisirs.

Les quotidiens japonais sont caractérisés depuis longtemps par leur fort taux de pénétration. Ainsi, le Yomiuri shinbun 読売新聞 et l'Asahi shinbun 朝日新聞, journaux au centre de notre étude, constituent les deux premiers

\footnotetext{
\ Doctorant en études japonaises rattaché à l'IETT (université Lyon 3), Tino Bruno s'intéresse au nucléaire civil et militaire, à son image, ainsi qu’à sa promotion au sein des médias de masse.

1. Pour reprendre les termes prononcés par le Premier ministre japonais de l'époque, Kan Naoto 管直人, lors d'un discours officiel le 13 mars 2011.

2. Voir l'article de Thierry Ribault, "L'empire du nucléaire : quand Fukushima ne crachait pas encore ses "atomes pour la paix" " dans le présent numéro [N.D.L.R].
} 
tirages mondiaux. Il n'y a alors rien d'étonnant à ce qu'ils aient constitué un excellent terreau pour la propagande étatique. Aussi, les liens étroits entre personnalités politiques, propriétaires de journaux et grands groupes énergétiques ont facilité l'introduction et l'acceptation du nucléaire civil au sein de la société japonaise.

Si la promotion du nucléaire civil dans la presse constitue un cas d'étude intéressant, force est de constater que la littérature sur ce sujet n'est pas très abondante ${ }^{3}$.

C'est en partant de ce constat que nous essayerons, dans cette contribution, de présenter de manière brève, et à travers quelques exemples concrets, quels ont été les trois principaux "mythes " entretenus par la presse dans une logique de promotion du nucléaire : le mythe du nucléaire de paix, le mythe de la sûreté et enfin le mythe de la nécessité. En conclusion, nous nous interrogerons sur les répercussions qu'ont pu avoir les accidents nucléaires sur l'évolution de ces mythes, notamment dans une société japonaise traumatisée par l'accident nucléaire de Fukushima et où les nouveaux médias fleurissent.

\section{Mythe du nucléaire de paix}

Alors que le souvenir de la bombe atomique marquait encore les esprits, les médias japonais ont, sur le modèle américain, séparé l'atome en deux champs d'application dès la fin de la Seconde Guerre mondiale. On parlait alors de gunji riyo 軍事利用 et de heiwa riyo 平和利用 pour désigner respectivement le nucléaire militaire et le nucléaire civil. En effet, tout en soulignant son aversion pour le nucléaire militaire, la presse quotidienne japonaise insistait sur les possibilités offertes par son pan civil et sur la situation unique dans lequel se trouvait le Japon, "seul pays ayant subi la bombe atomique au monde ${ }^{4}$ ", et de l'opportunité que constituait le «nucléaire de paix » pour lui ${ }^{5}$.

3. Notre bibliographie constitue toutefois une première piste pour celui qui veut en savoir davantage sur le sujet.

4. Pour reprendre l'expression japonaise «sekai de yuiitsu no hibaku koku 世界で唯一の 被爆国 ", popularisée après-guerre.

5. Le nucléaire était aussi au cœur d'une stratégie constituant à tourner rapidement la page de l'après-guerre, laquelle rappelait la défaite technologique subie, et cela passait 
Dès le 10 septembre 1947, l'Asahi shinbun titrait "Genshiryoku no heiwateki riyō » 原子力の平和的利用 (L'utilisation pacifique du nucléaire) et revendiquait un nucléaire de paix, au lendemain d'un discours du Président américain Harry S. Truman prônant le nucléaire civil.

Si les débuts de la couverture du nucléaire civil ont été timides (Nakamura 2005), les médias japonais commencèrent vraiment à promouvoir l'énergie à la suite du discours "Atoms for Peace ". À ce titre, Shōriki Matsutarō 正力松太郎 (1885-1969), ancien propriétaire du quotidien Yomiuri shinbun mais aussi premier Genshiryoku iinkai iinchō 原子力委員会委員長 (président de la Commission de l'énergie atomique) dès 1956, joua un rôle non négligeable dans la promotion du nucléaire au Japon ${ }^{6}$. Dès le début des années 1950, et avec l'appui du gouvernement américain et de certaines personnalités américaines, notamment de la CIA (Arima 2008), il embarqua son journal dans une campagne de promotion du nucléaire dit " de paix ». On retiendra notamment la création de la "Bourse Yukawa ", la série d'articles intitulée "On a enfin pu saisir le soleil ${ }^{8}$ » en 1954 ou encore l'organisation d'une "Exposition à propos du nucléaire de paix » avec la participation de l'United States Information Service dès l'année suivante (Ikawa 2002, Tanaka 2011). Le retentissement de cette exposition eut raison des trente millions de signatures réclamant un bannissement du nucléaire, qu'avait soulevé peu de temps auparavant l'accident nucléaire du Daigo Fukuryū-maru 第五 福龍丸. Ce bateau avait été contaminé par l'essai nucléaire de Castle Bravo au large de l'atoll de Bikini le $1^{\text {er }}$ mars 1954, et ses vingt-trois membres d'équipage furent irradiés, dont Kuboyama Aikichi 久保山愛吉, lequel mourut quelques mois plus tard. L'équipe médicale japonaise déclara

par une excellence technologique et économique.

6. Ce dernier put notamment compter sur l'aide de Nakasone Yasuhiro 中曽根康弘 (1918-) ou encore de Tanaka Kakuei 田中角栄 (1918-1993), deux hommes politiques japonais occupant des places stratégiques à l'époque.

7. Du nom de Yukawa Hideki 湯川秀樹 (1907-1981), prix Nobel de physique (1949). Ce dernier jouera un rôle important dans la recherche nucléaire au Japon.

8. “Tsui ni taiyō o toraeta »ついに太陽をとらえた est une série de trente et un articles diffusée dans le Yomiuri shinbun du $1^{\text {er }}$ janvier au 9 février 1954 et supervisée par Nakamura Shintarō 中村誠太郎, élève de Yukawa Hideki, dans le but de familiariser la population à la technologie nucléaire. 
qu'il était décédé d'un syndrome d'irradiation aiguë, ce que les autorités américaines refusèrent et refusent encore à ce jour de reconnaître.

Selon Shibata Tetsuji 柴田鉄治9 ${ }^{9}$ cet accident encouragea fortement les journaux à mettre en place une section "sciences " au sein de leur rédaction (Nihon genshiryoku sangyō kaigi 2004: 151). Le Yomiuri shinbun qui avait, le premier, révélé l'accident nucléaire fera figure de précurseur (1956) alors que son concurrent l'Asahi shinbun ne le rejoindra qu'un an plus tard.

La même année, le Genken Tōkaimura kenkyūjo 原研東海村研究所 (Réacteur nucléaire expérimental de Tōkaimura) accueillera la première réaction en chaîne du Japon, laquelle sera reçue de façon très favorable par les journaux de l'époque, l'Asahi shinbun parlant même d'une " date mémorable pour le Japon ${ }^{10}$ ».

\section{Mythe de la sûreté}

Le mythe de la sûreté ${ }^{11}$ apparaît dans les années 1950 et fut entretenu à mesure que l'énergie se rapprochait de la population civile à travers les différentes étapes de l'aventure nucléaire : décision d'une politique énergétique, recherche d'emplacements pour les centrales, construction et mise en service de ces dernières (la première entrera en fonction en 1966). Ce mythe de la sécurité reposait sur trois piliers : une technologie japonaise d'excellence, un partenaire américain expérimenté et rassurant et une conception des risques - notamment naturels - propre au Japon. À ce titre, un article publié le 10 mai 1959 dans l'Asahi shinbun éclaire parfaitement ce mythe : un spécialiste américain y complimente l'excellence de la technologie

9. Shibata Tetsuji fut journaliste à l'Asahi shinbun à partir de 1959. Il deviendra par la suite directeur de la section scientifique du journal.

10. Encadré “Yuwakashi-gata wa sekai de kyūban me "湯沸し型は世界で九番目 (Neuvième réacteur à eau bouillante au monde) au sein de l'article " "Genshiryoku no hi” Nihon ni hajimete tomoru »「原子力の火」日本に初めてともる (Le « feu nucléaire ” allumé pour la première fois au Japon), 27 août 1957, Asahi shinbun.

11. On parle d'anzen shinwa 安全神話 en japonais. 
japonaise $^{12}:$ "Les chercheurs japonais sont très compétents et excellents dans leurs décisions, ils produiront dans un avenir proche des résultats tout aussi excellents "; et prête son expertise pour affirmer que les projets japonais ne sont en aucun cas dangereux : «Des gens se demandent s'il n'est pas dangereux d'installer plus d'une dizaine de réacteurs nucléaires dans une zone aussi étroite que Tōkaimura, en fait cela n'a rien de préoccupant ${ }^{13}$ ".

Un article publié le 18 février 1961 dans le Yomiuri shinbun se veut encore plus convaincant en insistant sur le caractère psychologique de la peur du nucléaire au Japon et en louant le caractère exceptionnel des critères de sécurité japonais, notamment à propos des mesures parasismiques :

"De même que des critères de sécurité sans égal dans le monde vont être suivis systématiquement lors de la construction des centrales et des machines, ils vont également être suivis au sein de l'industrie nucléaire et par là même lui donner une ligne de conduite. Notre peur du nucléaire provient d'un sentiment ancré au sein de la population depuis le bombardement atomique de Hiroshima. [...] Bref, si l'on suit ces critères à la lettre, même si un séisme d'amplitude prévue venait à survenir, cela ne serait pas un problème. C'est vraiment "la sécurité avant tout"14. "

Aussi, dès 1964 apparaît la "Genshiryoku no hi » 原子力の日 (Journée du nucléaire) durant laquelle de nombreuses manifestations permettent de "désacraliser " l'atome civil auprès de la population et d'atténuer sa peur du nucléaire. C'est d'ailleurs à l'occasion de la deuxième " Journée du nucléaire " que le quotidien Asahi shinbun publia en une de son édition du soir un article parlant du réacteur nucléaire comme d'un " outil qui n'est déjà plus ni rare ni dangereux ${ }^{15}$ ».

12. Asahi shinbun, 10 mai 1959, "Tōkaimura ni kiken ha nai : genken keikaku de Wa hakushi kataru ” 東海村に危険はない——原研計画でワ博士語る (Aucun danger à Tōkaimura : le professeur Alvin M. Weinberg parle du projet de centrale expérimentale).

13. Ibid.

14. Yomiuri shinbun, 18 février 1961, "Genshiryoku hatsudensho no anzen kijun : subete anzen daiichi shugi » 原子力発電所の安全基準——ずて安全第一主義 (Les critères de sécurité des centrales nucléaires : politique du "sécurité avant tout ").

15. Asahi shinbun, 26 octobre 1965, encadré «Konnichi no mondai » 今日の問題. 


\section{Mythe de la nécessité}

En toute logique, et en contrepoids essentiel aux peurs liées au nucléaire militaire et à celles d'un accident industriel, le mythe de la nécessité apparaît à peu près au même moment que les deux autres. Il prend appui sur les arguments suivants : le défi technologique, la dépendance énergétique d'un pays pauvre en ressources naturelles puis la recherche d'une énergie propre au moment où les questions d'environnement seront posées.

Comme l'observe Itō Hiroshi 伊藤宏, l'Asahi shinbun souligne déjà la nécessité du nucléaire face au manque de ressources dans cet article à la une de l'édition du 16 avril 1955 :

"L'utilisation de la nouvelle énergie nucléaire suit une logique mondiale, et plus particulièrement pour notre pays pauvre en ressources énergétiques, il ne fait aucun doute qu'elle constitue une lueur d'espoir pour l'avenir ${ }^{16}$."

Alors que les critiques du nucléaire se font de plus en plus remarquées dans la presse quotidienne japonaise des années 1960, deux éléments viennent renforcer les arguments des pro-nucléaires au Japon : le problème de la pollution environnementale et le premier choc pétrolier. À partir de la fin des années 1960, la question environnementale, notamment portée par le scandale de Minamata-byō 水俣病 (maladie de Minamata ${ }^{17}$ ), émerge au Japon et aboutit en 1970 à la création du Kankyōchō 環境庁 (agence de l'Environnement) alors qu'en 1973 le premier choc pétrolier crée la panique dans un pays encore peu nucléarisé et très dépendant des pays pétroliers.

Au moment où le Japon se lance dans l'énergie solaire ${ }^{18}$, les médias japonais mobilisent à nouveau les thèmes fondateurs du mythe de la nécessité pour promouvoir l'atome, et insistent cette fois sur les termes de kurin enerugī クリーンエネルギー, énergie propre, et de kosuto yasu コスト安, coût bas.

16. Asahi shinbun, 16 avril 1955, "Ze ka hi ka : Nichi-Bei genshiryoku kyōtei " 是 か非か一一米原子力協定 (Pour ou contre l'accord bilatéral nippo-américain sur le nucléaire).

17. Voir les travaux de Paul Jobin sur le sujet, notamment Maladies industrielles et luttes syndicales au Japon (2006).

18. Au sein du «Sanshain keikaku » サンシャイン計画 (Plan «Soleil ») lancé en 1974, à l'occasion duquel le gouvernement japonais investira des sommes astronomiques afin de promouvoir de nouvelles énergies propres. 
Par exemple au journal Asahi shinbun, cette promotion sera menée entre autres par des journalistes tels que Ōkuma Yukiko 大熊由紀子, auteure d'une série de quarante-huit articles publiée du 13 juillet au 5 septembre 1976 intitulée "Kakunenryō : tansa kara haikibutsu shori made " 核燃 料一一探査から廃妄物処理まで (“Combustible nucléaire : de l'exploration au traitement des déchets»). Kishida Junnosuke 岸田純之助, initateur du slogan «Yes, but ", prônant le nucléaire tout en prenant en compte les risques qui y sont liés, prêtera son concours à la même entreprise.

\section{Conclusion}

Cette contribution tente de décrypter la promotion du nucléaire dans la presse quotidienne japonaise, en se focalisant sur la période allant de l'après-guerre jusqu'à la fin des années 1970, période qui verra naître les " mythes du nucléaire " et sera témoin de leur heure de gloire. L'année 1979 apporte en effet son lot de bouleversements : deuxième choc pétrolier, accident à la centrale de Three Mile Island, indices d'instabilité sur des installations japonaises, amorce d'une sortie du nucléaire civil en Suède. Autant de facteurs qui fragilisèrent la promotion du nucléaire, l'accident de Tchernobyl (1986) et les accidents survenus au Japon dans les années 1990-2000 « aggravant » encore la situation.

Jusqu'à peu, la société civile ne pouvait pratiquement que se fier aux médias traditionnels, auxquels elle accordait un crédit sans réserve. La manière dont la société japonaise fait face à la catastrophe du 11 mars 2011 et au désastre nucléaire montre toutefois l'importance que jouent les nouveaux médias. Si la méconnaissance du public des sujets techniques tels que le nucléaire a pu faciliter sa promotion à l'époque, elle peut aussi être à l'avenir exploitée par les nouveaux médias dans le but contraire. Ces médias sont de fait devenus des outils permettant d'échapper aux circuits traditionnels de l'information dont nombre de Japonais se méfient de plus en plus, notamment à cause des pressions économiques exercées par les grands groupes énergétiques, qui comptent parmi les plus grands diffuseurs publicitaires. On peut faire l'hypothèse que la diffusion de l'idée d'un « effondrement du mythe de la sécurité » doit beaucoup à ces nouveaux médias citoyens.

30 novembre 2011 (finalisé le 14 février 2012) 


\section{Bibliographie}

ARIMA Tetsuo 有馬哲夫, 2008

Genpatsu, Shōriki, CIA : Kimitsu

bunsho de yomu Shōwa rimen-shi

原発・正力·CIA 一機密文書で読吉昭和裏

面史 (Centrales nucléaires, Shōriki, CIA :

les dessous de l'ère Shōwa à travers la

lecture de documents secrets ), Tokyo,

Shinchōsha 新潮社.

IKAWA Mitsuo 井川充雄, 2002

«Genshiryoku heiwa riyō hakurankai to shinbunsha » 原子力平和利用博覧会と 新聞社 (Les expositions à propos de I'utilisation pacifique du nucléaire et les journaux), in TSUGANESAWA Toshihiro 津金沢聡広 (dir.), Sengo Nihon no media ibento 戦後日本のメディアイベント (Les événements médiatiques du Japon d'après-guerre), Kyoto, Sekai shisō sha 世界思想社, pp. 248-265.

ITŌ Hiroshi 伊藤宏, 2004-2009 «Genshiryoku kaihatsu riyō o meguru media gidai : Asahi shinbun shasetsu no bunseki » 原子力開発・利用をめぐ るメディア議題: 朝日新聞社説の分析 (Agenda-setting des médias au regard du développement et de l'utilisation de l'énergie nucléaire : analyse des éditoriaux du journal Asahi shinbun) in Pūru gakuin daigaku kenkyū kiyō プール学 院大学研究紀要, 44 : 63-76, 45 : 111-126, 49: 101-116.

JOBIN Paul, 2006

Maladies industrielles et luttes syndicales au Japon, Paris, EHESS.
NAKAMURA Isao (dir.) 中村功, 2005 Nihonjin no anzenkan kenkyū hōkokusho 日本人の安全観研究報告書 (Rapport de recherche sur la vision de la sécurité des Japonais), Tōyō daigaku 東洋大学.

\section{Nihon genshiryoku sangyō kaigi 日本原子力産業会議, 2004 Genshiryoku nenkan 2005 原子力年鑑 2005 (Almanach du nucléaire 2005), Tokyo, Nihon genshiryoku sangyō kaigi 日本原子力産業会議.}

TANAKA Toshiyuki 田中利幸, 2011 " "Genshiryoku heiwa riyō" to Hiroshima : senden kōsaku no tāgetto ni sareta hibakusha tachi »「原子力平 和利用」とヒロシマ——宣伝工作のターゲ ットにされた被爆者たち (Hiroshima et I'« utilisation pacifique du nucléaire »: lorsque les irradiés étaient la cible de la propagande), in TANAKA Toshiyuki \& KUZNICK Peter, Genpatsu to Hiroshima. "Genshiryoku heiwa riyō » no shinsō 原発とヒロシマ—「原子力平和利用」の 真相 (Centrales nucléaires et Hiroshima. La vérité sur l'« utilisation pacifique du nucléaire »), Tokyo, Iwanami shoten 岩波書店, pp. 23-61. 